\title{
La capacitación laboral como herramienta de mejoramiento empresarial
}

\section{Job training as a tool for business improvement}

\author{
Byron Medina Delgado ${ }^{1}$ \\ Wlamyr Palacios Alvarado ${ }^{2}$ \\ Mawency Vergel Ortega ${ }^{3}$ \\ UFPS
}

\section{RESUMEN}

El factor de capacitación en un mundo en donde la economía del conocimiento incrementa su relevancia como fuente de ventaja competitiva, es fundamental para el éxito organizacional y del país. La investigación tiene como objetivo evaluar la capacitación laboral en la mediana empresa de Cúcuta-Colombia. La evaluación de la capacitación laboral se fundamenta en la caracterización de los indicadores de educación media y superior, capacitación empresarial y capacidades administrativas, mediante la aplicación de un instrumento a 138 empresas. En los resultados se evidencia que el $92 \%$ de

$1 \quad$ Universidad Francisco de Paula Santander, Cúcuta, Colombia. Docente, Investigador https://orcid.org/0000-0003-0754-8629 byronmedina@ufps.edu.co

2 Universidad Francisco de Paula Santander, Cúcuta, Colombia. Docente, Investigador https://orcid.org/0000-0002-4292-4178 wlamyrpalacios@ufps.edu.co

3 Universidad Francisco de Paula Santander, Cúcuta, Colombia. Docente - Investigadora https://orcid.org/0000-0001-8285-2968 mawencyvergel@ufps.edu.co la mediana empresa es gerenciada por una persona con título profesional y el $74 \%$ de las organizaciones promueven el trabajo en equipo como estrategia para concentrar habilidades, destrezas, conocimientos y responsabilidades. Se concluye que la mediana empresa de Cúcuta les apuesta a buenos indicadores con respecto a la educación media y superior, la capacitación empresarial y las capacidades administrativas, considerándolos herramientas fundamentales para el mejoramiento del sector empresarial.

\section{PALABRAS CLAVES:}

Capacitación laboral, mediana empresa, organización, recurso humano, trabajo en equipo.

\section{ABSTRACT}

The training factor, in a world where the knowledge economy increases its relevance as a source of competitive advantage, is essential 
for organizational and country success. The investigation aims to evaluate job training in a medium-sized company in Cúcuta, Colombia. The evaluation of job training is based on the characterization of the indicators of secondary and higher education, business training and administrative skills, through the application of an instrument to 138 companies. The results show that $92 \%$ of the medium-sized company is managed by a person with a professional degree and $74 \%$ of the organizations promote teamwork as a strategy to concentrate abilities, skills, knowledge and responsibilities. It is concluded that the medium-sized company in Cúcuta, bets on good indicators with respect to secondary and higher education, business training and administrative capacities, considering them fundamental tools for the improvement of the business sector.

\section{KEYWORDS:}

Job training, medium-sized business, organization, human resources, teamwork.

\section{INTRODUCCIÓN}

La investigación evaluar y para ello identificar mejoras y procesos con baja calidad en las empresas medianas que se encuentran en la ciudad de Cúcuta, Norte de Santander, cuando están en su operación básica; esto, enfocado en la capacitación como pilar de desarrollo y progreso para cada una de las empresas, las cuales generan preguntas como ¿Es necesario capacitar el personal?, ¿es necesaria la inversión para el desarrollo de los trabajadores al aportarle conocimiento mediante las capacitaciones?, todo se verá desde el punto de vista empresarial, administrativo y educativo. Muchas empresas frecuentan dificultades frente a otras que la hacen más vulnerables al no poder proveerse del conocimiento y de las capacidades necesarias para su crecimiento, esto por la falta de recursos que es la causa principal por la cual muchas empresas no pueden posicionarse en el mercado, como tal la capacitación da una visión general de la empresa para poder construir su futuro, en el momento de operar negocios, tener responsabilidades y todas las exigencias que conlleva el mercado. El ámbito de estudio abarca la ciudad de Cúcuta, Norte de Santander, con respecto al desarrollo de estrategias de capacitación, aunque es preciso identificar sus necesidades y concluir mejoras para el funcionamiento de las medianas empresas de la ciudad, en cuanto al proceso de desarrollo del artículo se tomó información recolectada por medio de un instrumento. Mondy, R \& Noe (2005), señalan la importancia de la capacitación de los recursos humanos en la empresa para el desarrollo de ésta, aunque resaltan que se puede deducir que son importantes las evaluaciones de cada aspecto dentro de la labor y la competitividad que se pueda desarrollar dentro de la compañía, permitiéndole tener una mejora continua tanto para la misma, como para el personal humano que hace parte de su cobertura.

Así mismo, se busca evaluar resultados respecto a la mejora de la capacitación en las empresas medianas, pero también se analizan factores tales como la influencia del trabajo en equipo, personal capacitado dentro de la empresa y la importancia de capacitar el personal ya sea con recursos propios de la empresa o con recursos externos; por ende es muy importante el uso de un instrumento aplicado en la mediana empresa de la ciudad de Cúcuta, Norte de Santander, buscando por medio de éste analizar mejoras para la capacitación laboral como herramienta de mejoramiento.

En capacitación, en la organización de las medianas empresas genera un valor agregado tanto a los empleados como a la compañía, ya que genera competitividad y distintivas sobre otras, por ende, se hace necesario la capacitación de cada escenario para transmitir a los empleados el conocimiento y la aplicabilidad a cada una de las áreas de la compañía. (Silíceo, 
1993). Para tal fin en educación media y superior, una economía competitiva requiere articular la educación media con la superior; articulación decisiva para el desarrollo de competencias laborales y la formación de recurso humano calificado, enfocadas a los sectores claves para el progreso del país. Con el apoyo de la educación media y superior, se deben fortalecer la comprensión de lectura, la expresión oral y escrita, y la competencia del idioma inglés (Consejo Privado de Competitividad, 2017), se plantea que, en la actualidad, el capital intelectual se reconoce como la fuente fundamental de la verdadera y única ventaja competitiva que se sostiene en una comunidad, el conocimiento como tal es un recurso básico del humano por lo cual lo tenemos presente en la sociedad (Marr, 2004). Se podría conocer como el grupo de conocimiento que se le otorga a una comunidad. El recurso humano con más experiencia en la empresa o con alto conocimiento, es aquel que tiene conocimiento y puede generar innovación, un personal preparado y con un alto conocimiento en sus obligaciones dentro de la empresa es el que puede generar una competitividad en el mercado y en la empresa por hacer mejor las cosas (Colciencias, 2005). Por tal motivo, es importante que desde las diferentes regiones sigan incentivando a convocatorias para formar el personal a tener un alto nivel, a ser especialistas, magísteres y doctores, mediante el apoyo del gobierno a través de becas con entidades nacionales, incluso las pasantías de investigadores en empresas, para generar una interacción entre la academia y el sector productivo de la empresa, este apoyo podría garantizar un desarrollo competitivo a nivel regional y nacional (Colciencias 2005).

En este contexto las universidades son fundamentales en el desarrollo competitivo de las empresas y tienen la labor de construir un indicador que permita dimensionar el potencial de las cadenas productivas estratégicas de las regiones, de generar dinámicas de ciencia, tecnología e innovación, a partir de la medición de variables como generación de valor, encadenamientos productivos, requerimientos de formación especializada del recurso humano, generación de conocimiento, orientación exportadora, atracción de inversión, entre otros (Colciencias, 2014).

Según el contexto actual el cual se ve influenciado por un cambio tecnológico constante, la capacitación en la empresa, en el ámbito del personal tiene un papel fundamental para generar competitividad, por un lado, complementa la educación formal porque posibilita al trabajador apropiar los conocimientos y habilidades requeridas para utilizar la tecnología, adaptarla y eventualmente mejorarla. Asimismo, al estar orientada a proveer los conocimientos y habilidades que los empleados exigen para sus actividades diarias, es posible pensar que signifique rápidas y valiosas utilidades para la empresa. Para que una organización conserve su competitividad en un ambiente de constantes cambios en preferencias y tecnologías, se requieren trabajadores capaces de adaptarse a los cambios y de innovar apresuradamente. Con esto se busca generar un valor al personal de las empresas, por medio de la capacitación empresarial, dando como resultado conocimiento y nuevas habilidades que le permiten desempeñarse mejor en su lugar de trabajo y a su vez generando una competitividad necesaria para la mejora continua, generando un crecimiento de la compañía. En otro contexto según (Gallart, 2001). Es indispensable reconocer que existen dos tipos de capacitación en la empresa: la capacitación general aplicable en más de una empresa y la capacitación específica asociada con habilidades y conceptos propios de una empresa. Las organizaciones, por la dificultad de apropiación de los resultados de la formación, tienden a invertir en esta última. Esto quiere decir que la capacitación puede variar, unas pueden ser aplicadas en diferentes empresas ya que van enfocadas a temas 
generales y otras capacitaciones se centran como tal al conocimiento interno de la compañía, por ende, solo puede ser reproducida esta información de la capacitación dentro de cada empresa. La probabilidad de que las empresas decidan capacitar a sus empleados varía en los recursos de la misma, ya sea el tamaño y la cobertura con el que cuenta la empresa, los niveles de educación de la empresa, o los necesarios para ejercer sus puestos, el que la empresa quiera tener nuevas tecnologías que le ayuden en sus procesos, los controles de calidad y el capital extranjero, según "Padilla y Juárez, 2007". Investigaciones de países en desarrollo han sustentado que la capacitación en el trabajo brinda significativos retornos en términos de productividad. Sin embargo, un número relevante de empresas de manufactura y servicios, especialmente las medianas, no brindan algún tipo de capacitación formal (Batra y Tan, 2002). Podemos entonces concluir que muchas de las causas de la ausencia de capacitación dentro de la mediana empresas se deben a la falta de información relacionada con los beneficios que podrían obtener al realizar estas inversiones, se puede deducir sobre la disminución de altos costos dentro de la empresa, por eso en empresas con alto índice de rotación del personal, no es común encontrar una cultura de capacitación ya que se toma en cuenta que estos pueden luego ser contratados por otras empresas. Como tal, las capacitaciones pueden desarrollarse con recursos internos o externos, generalmente las capacitaciones con recursos internos se dan por medio de los jefes, supervisores o con otros compañeros, los cuales la misma empresa busca un salón para realizarlas y en sus horarios de trabajo establecidos; y las capacitaciones con recursos externos como tal se desarrollan por medio de la contratación de personas especializadas en los temas que necesita abordar o mejorar la empresa, estas consultas pueden realizarse con proveedores, gremios empresariales o universidades que tenga el personal debidamente calificado para poder capacitar de una manera adecuada al personal.

Una definición de capacidades administrativas como ese conocimiento que obtiene la empresa que le resulta como experiencia que le permite a los jefes o líderes de la empresa afrontar con carácter, decisión y sabiduría todos los desafíos que se plantean en los diferentes ámbitos de la empresa, siendo estas habilidades un aporte para el crecimiento y la mejora continua de la organización (Arocha, 2016) Por lo tanto, Colmenares y Villasmil (2008) proponen tanto para el líder como el gerente el requerimiento de ser competentes, hábiles, para enfrentar la incertidumbre del entorno, operando eficazmente ante los problemas que se presenten; con lo que quiere decir el conocimiento que se le da al líder para enfrentar todo tipo de situaciones que se le requieran sean solucionadas por este.

Por ende, para Pérez (2004) en la actualidad las organizaciones requieren personas con capacidades que incrementen la creatividad y flexibilidad, para tomar decisiones efectivas $y$ adaptables a diferentes culturas. Dicho esto, se puede observar la importancia que tiene el capital humano preparado y adecuado para que estos incrementen el conocimiento adecuado para una toma de decisiones efectiva y que se adapte a las necesidades de cada tiempo de la empresa.

La toma de decisiones es una conducta que puede solucionar problemas en diferentes situaciones muchas inciertas, por eso se basa en el análisis del contexto propio de la situación y fijar aquellos componentes respectivos, con el fin real de determinar un plan para proponer las correcciones necesarias (Arocha, 2016). Para "Sánchez, 2006" el trabajo en equipo concentra un grupo de personas para lograr metas comunes y la toma de decisiones, integrando sus conocimientos y habilidades; este trabajo en equipo es fundamental en toda 
organización, e implica el compromiso de todos los integrantes. El trabajo en equipo pretende un objetivo común y concentra las habilidades, destrezas, conocimientos y responsabilidades de todos los integrantes. Según la información anterior estas sugieren que, para poder trabajar en equipo, se requiere integrar todo tipo de habilidades y responsabilidades que conlleven a la cooperación, al apoyo, a la comunicación entre el equipo y enfocar todas las habilidades en un objetivo común, dando como resultado un rendimiento mayor y siendo una empresa competitiva y exitosa en su campo económico.

La motivación está ligada al interés de lograr el cumplimiento de las metas propuestas por la organización, siendo esto un pilar para abrir las puertas de la competitividad en los mercados (Robbins \& Coulter, 2005).

\section{METODOLOGÍA}

La perspectiva acogida concierne con el enfoque positivista, conocido también como empíricoanalítico, científico-técnico, cuantitativo o racionalista. Tal como lo señalan Hernández, Fernández \& Baptista (2010), este se utiliza en la recolección de datos que posteriormente responden a las preguntas formuladas en la investigación. En esta propuesta se obtiene información sobre la capacitación empresarial en la mediana empresa de Cúcuta, Colombia, representa un tipo de visión que orienta, una perspectiva teórica que es aceptada por la comunidad científica, que establece y/o determina qué estudiar y por consiguiente formula hipótesis explicativas de los fenómenos observados (Corbetta, 2007; Toro, 2008 Palella \& Martins, 2012; Boza 2012) Según Ramírez (2009) el paradigma científico-técnico, surge con la extensión de las ideas propias de las ciencias naturales a las ciencias sociales, no haciendo distinción entre dichos fenómenos. En ese sentido para Terán (2006) la teoría positivista aspira establecer las causas de los fenómenos sociales presentando generalidades acerca de las observaciones. Si la realidad está en el exterior, es observable, medible y cuantificable; es decir, es determinada.

Para Palella \& Martins (2012) este paradigma se centra en la operacionalización, en la descomposición del todo y en su posterior integración. En este sentido la percepción humana se minimiza a factores medibles, analizables, y luego a través de la estadística se asigna la probabilidad de ocurrencia, convirtiéndolo en la esencia de la investigación cuantitativa.

En esta perspectiva, la postura epistemológica de esta investigación, se centra en el paradigma positivista, porque considera el marco conceptual (comprobación empírica), las técnicas de observación y medición (uso de variables cuantitativas), los instrumentos de análisis matemático y los procedimientos de inferencia de las ciencias naturales (Corbetta, 2007). Adicionalmente, esta investigación asume de los paradigmas neopositivista y postpositivista, que las teorías sociales no se deben expresar en forma de leyes deterministas, sino en términos probabilísticos, centrándose en el lenguaje de las variables, procedente de las matemáticas y la estadística.

Esta investigación es descriptiva con dos variables, no experimental, transaccional y de campo. La investigación descriptiva radica en conocer las realidades, costumbres y actitudes sobresalientes, a través de la descripción exacta de las actividades, objetos y procesos con las personas. Este tipo de investigación describe lo que existe, aportando al descubrimiento de nuevos hechos y significados. De acuerdo con Tamayo \& Tamayo (2003, p.46) la investigación de tipo descriptiva opera sobre realidades 0 hechos, con el fin de presentar la acertada interpretación; involucrando la recolección de datos que representen a los individuos, grupos o situaciones. Los instrumentos usados para adquirir datos en los estudios de tipo descriptivo 
incorporan instrumentos como cuestionarios, entrevistas (preguntas cerradas) y observación (listas de verificación). No existe manipulación experimental en estos grupos.

Para Arias (2006) el nivel descriptivo representa la profundidad del estudio, calculando las variables independientemente. De igual manera, no se plantean hipótesis porque están referenciadas en los objetivos de la investigación. Adicionalmente, para Méndez (2007), el carácter descriptivo caracteriza el universo de estudio. Esto asocia las variables estudiadas, manifestando la estructura del problema. Para esto se utilizan técnicas concretas con criterios sistemáticos, correspondiendo las variables sin crear relaciones de causalidad entre éstas. En este contexto para Palella \& Martins (2012) establecen que la investigación de tipo descriptiva descifra hechos y realidades; detallando, registrando, analizando y descifrando las características y los factores en el objeto de estudio. En resumen, el tipo descriptivo se enfoca en determinar los factores del momento actual. El estudio pretende diagnosticar el hecho vigente en la mediana empresa de la ciudad de Cúcuta, Colombia.

Para esta investigación, se implementó el método no experimental porque no se manipuló el contexto en donde se realizó el estudio. Las variables son medidas y registradas de acuerdo con la información recolectada con los instrumentos. De igual manera, se determinan características en las investigaciones no experimentales, como la presentación real del fenómeno a como se expresa en su entorno; es decir, sin la manipulación de las variables. Además, las investigaciones descriptivas aportan más conocimiento al condensar trabajos de diferentes áreas del conocimiento, que se pueden enfocar a un objeto en común. La investigación es transeccional porque los factores y las variables objeto de estudio, se miden en un solo momento del tiempo.
En este sentido se encaminan considerando que la investigación estudia la relación en un período específico. Según Arias (2006) el diseño transeccional o transversal es aquel cuya información recolectada sólo tiene validez en el periodo que se obtiene; porque ésta puede variar con el tiempo. Adicionalmente, el instrumento está relacionado con el tipo de investigación, por lo tanto, se entiende a la encuesta como investigación con diseño transversal o transeccional.

La investigación es de campo considerando la aplicación del instrumento al objeto de estudio. Según Arias (2006), la investigación de campo, consigue información sin modificar o alterar las circunstancias de las variables, aportando un carácter no experimental. Es en este escenario en donde la información no se perturba, porque los datos son recolectados sin manipulación alguna, involucran otras fuentes de información que no son bibliográficas, mediante métodos y técnicas aplicables en sitio, son provechosas Chávez (2007).

Para determinar la población, se clasificaron las organizaciones según los activos totales, de acuerdo con la clasificación de las empresas que plantea el ministerio de comercio, filtrando la base de datos de la Superintendencia de Sociedades (Supersociedades) de Colombia, para el departamento del Norte de Santander, en especial la ciudad de Cúcuta, y teniendo en cuenta las clasificaciones del Código Industrial Internacional Uniforme (CIIU). De acuerdo con este criterio, se obtuvo una población de 214. Clasificación de las empresas: Microempresa: aquella que cuenta con un personal que no supera los diez (10) trabajadores y cuyos activos totales por valor inferior a (500) salarios mínimos mensuales legales vigentes. Pequeña empresa: es una planta de personal que cuenta con un rango de empleados entre once (11) y cincuenta (50) trabajadores y sus activos totales por valor entre quinientos uno (501) y menos de cinco 
mil (5.000) salarios mínimos mensuales legales vigentes. Mediana empresa: una planta de personal entre cincuenta y uno (51) y doscientos (200) trabajadores y sus activos totales por valor entre cinco mil uno (5.001) a treinta mil (30.000) salarios mínimos mensuales legales vigentes. El cálculo sugirió para el estudio una muestra de 138.

En esta investigación se recurre al método de la encuesta y al cuestionario como instrumento Palella \& Martins (2012). De acuerdo con Méndez (2007) el cuestionario es un instrumento que permite recoger datos y que se aplica a una población seleccionada con anterioridad, de manera presencial o virtual mediante medios electrónicos. Según Bernal (2010) la encuesta es un conjunto de preguntas preparadas que se plantean en un documento y que permiten la obtención de información a partir de personas.

En el estudio se aplica un cuestionario estructurado, con nueve (9) preguntas cerradas, con una escala de Likert (Corbetta, 2007)., que pretende medir el nivel de apreciación del encuestado, con respecto al cumplimiento de las afirmaciones que se plantean de la siguiente manera: Nunca (opción 1), Casi nunca (opción 2), A veces (opción 3), Casi siempre (opción 4) y Siempre (opción 5). De acuerdo con lo anterior, se elaboró un baremo para la interpretación de las variables de estudio, el cual relaciona el grupo, el rango de los valores y el atributo, requeridos para el análisis del promedio, considerando las cinco (5) posibles. Según Mezher (2014), el valor numérico para las escalas se determina de la ecuación a continuación.

$$
R=(L s-L i) / n
$$

En donde $R$ es el rango utilizado para cada intervalo; $L s$ es el límite superior; $L i$ es el límite inferior; $y n$ es el número de atributos del baremo. Teniendo en cuenta que $L s$ y $n$ equivalen a 5 y $L i$ equivale a 1 , se obtiene rango de 0.8 para cada atributo; como muestra el cuadro 1.

Tabla 1. Baremo para interpretar la media

\begin{tabular}{ccc}
\hline Grupo & Rango de valores & Atributo \\
\hline 5 & $4.2 \leq R \leq 5.0$ & Muy bueno \\
\hline 4 & $3.4 \leq R<4.2$ & Bueno \\
\hline 3 & $2.6 \leq R<3.4$ & Regular \\
\hline 2 & $1.8 \leq R<2.6$ & Deficiente \\
\hline 1 & $1 \leq R<1.8$ & Muy deficiente \\
\hline
\end{tabular}

Fuente: Elaboración propia (2018)

La confiabilidad del instrumento propuesto se determinó con la técnica de Alfa de Cronbach Balestrini (2015), la cual mediante un coeficiente determina la confiabilidad entre 0 y 1 Corral (2009). Siete (7) expertos realizaron la prueba piloto al instrumento, dando un coeficiente de Cronbach de 0.91 interpretándose en rango 0.81 a 1 , es decir muy Alta; rango de 0.61 a 0.80 Palella y Martins (2006).

\section{RESULTADOS}

En las siguientes figuras y tablas, se verán los resultados del instrumento aplicado a las personas cuyo objetivo era evaluar los diferentes indicadores que interfieren en la capacitación, el resultado enfocado en el indicador de educación media y superior. Para evaluar este indicador el instrumento contó con 3 preguntas referentes: (1) ¿El gerente de la empresa tiene 
un título profesional?; (2) ¿La empresa tiene empleados que poseen solamente estudios de educación media?; (3) ¿Apoya la empresa a sus empleados para que cursen programas de educación superior?

Los resultados a estas preguntas se muestran en la figura 1 y tabla 1.

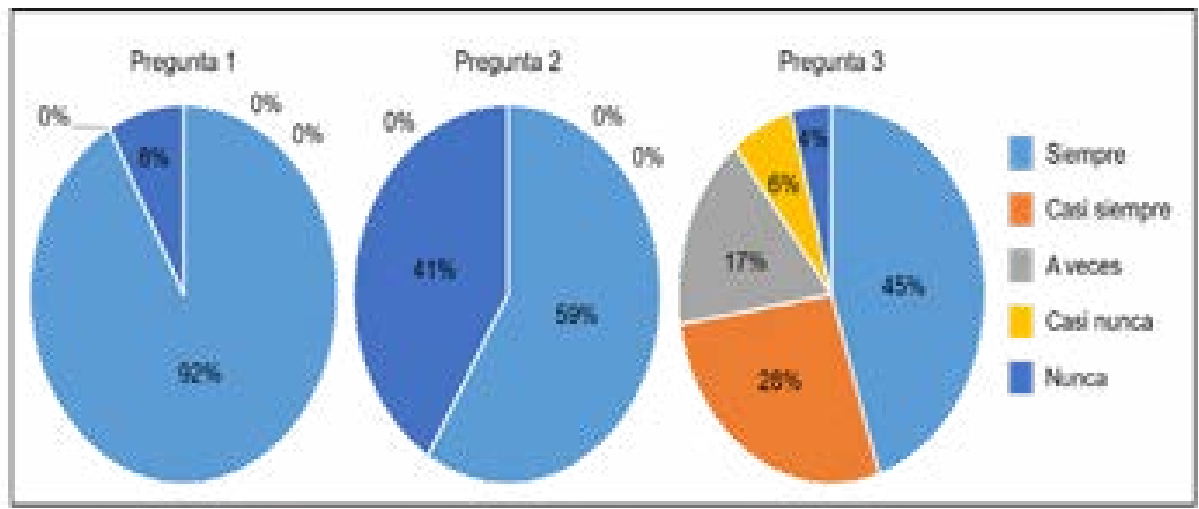

Figura 1. Respuestas de las preguntas 1, 2 y 3

Tabla 1. Indicador: educación media y superior

\begin{tabular}{|c|c|c|c|c|c|c|c|c|c|c|c|c|c|}
\hline \multirow{2}{*}{ Ítem } & \multicolumn{2}{|c|}{ Siempre } & \multicolumn{2}{|c|}{$\begin{array}{c}\text { Casi } \\
\text { siempre }\end{array}$} & \multicolumn{2}{|c|}{ A veces } & \multicolumn{2}{|c|}{ Casi nunca } & \multicolumn{2}{|c|}{ Nunca } & \multirow{2}{*}{ Total } & \multirow{2}{*}{ Atributo } & \multirow{2}{*}{$\sigma 2$} \\
\hline & $\%$ & Peso & $\%$ & Peso & $\%$ & Peso & $\%$ & Peso & $\%$ & Peso & & & \\
\hline 1 & 92,0 & 4,60 & 0,0 & 0,00 & 0,0 & 0,00 & 0,0 & 0,00 & 8,0 & 0,08 & 4,68 & $\begin{array}{l}\text { Muy } \\
\text { Bueno }\end{array}$ & 1,174 \\
\hline 2 & 58,7 & 2,93 & 0,0 & 0,00 & 0,0 & 0,00 & 0,0 & 0,00 & 41,3 & 0,41 & 3,34 & Regular & 3,879 \\
\hline 3 & 44,9 & 2,25 & 27,5 & 1,10 & 16,7 & 0,50 & 6,5 & 0,13 & 4,3 & 0,04 & 4,02 & Bueno & 1,268 \\
\hline \multicolumn{11}{|c|}{ Media del indicador } & 4,02 & Bueno & 0,296 \\
\hline
\end{tabular}

Como lo reflejan los resultados el indicador de educación media tiene una media de medias con valor de 4.02 lo cual podría valorarse como un resultado bueno, se puede deducir que tiene una varianza de 0.296 las cuales para estas respuestas fueron valoradas como muy bueno, regular y bueno.

Respecto a la primera pregunta del indicador, podemos observar que la mayoría de las empresas está siendo gerenciada por una persona con un título profesional, con un total del $92 \%$, y un número muy pequeño de empresas tan solo del $8 \%$ es gerenciada por una persona que no posee un título, siendo un resultado positivo en la mayoría. En la segunda pregunta se observó como resultado que más del $41 \%$ de la mediana empresa, tiene contratado mínimo un empleado cuyo nivel máximo educativo es el bachillerato.

En cuanto a la última pregunta podemos observar en el resultado que más del $95 \%$ de las empresas analizadas apoyan y promueven el estudio de programas educativos superiores por parte de sus empleados. En conclusión, 
se puede decir que la mediana empresa de la ciudad de Cúcuta considera relevante la cualificación de su personal.

Resultados enfocados en el indicador de Capacitación empresarial. Para evaluar este indicador el instrumento cuenta con 3 preguntas referentes: (4) ¿Realiza la organización capacitación específica asociada con habilidades y conceptos propios de la empresa?; (5) ¿Realiza la organización capacitaciones con recursos propios, a través de compañeros o instructores internos?; (6) ¿Realiza la organización capacitaciones con recursos externos, a través de consultorías expertas, proveedores de equipos o insumos especializados, gremios empresariales, universidades o instituciones técnicas? Los resultados a estas preguntas se muestran en la figura 2 y tabla 2.

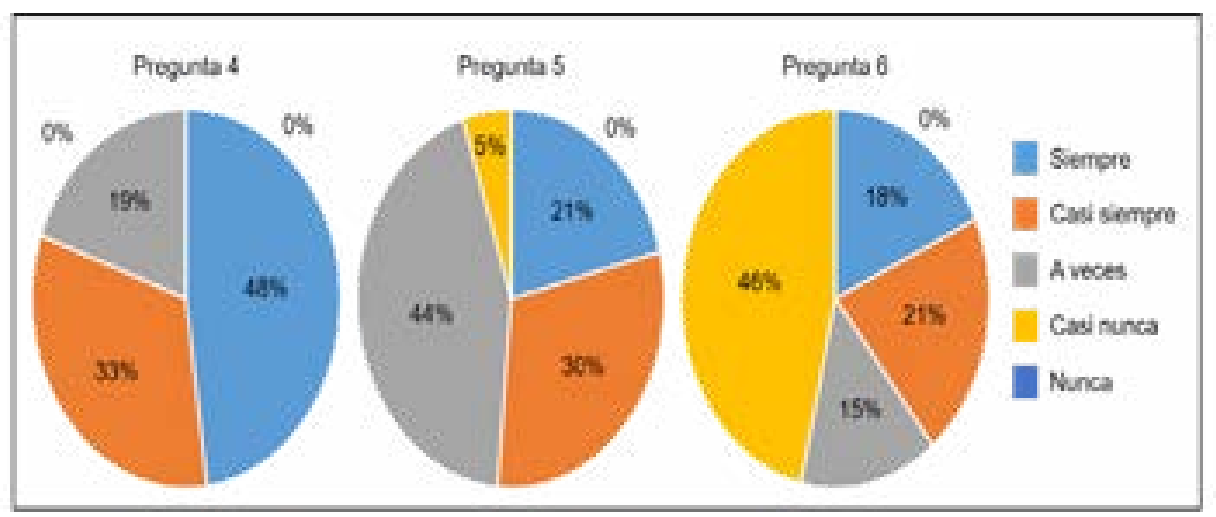

Figura 2. Respuestas de las preguntas 3,4 y 5

Tabla 2. Indicador: capacitación empresarial

\begin{tabular}{|c|c|c|c|c|c|c|c|c|c|c|c|c|c|}
\hline \multirow[t]{2}{*}{ Ítem } & \multicolumn{2}{|c|}{ Siempre } & \multicolumn{2}{|c|}{$\begin{array}{c}\text { Casi } \\
\text { siempre }\end{array}$} & \multicolumn{2}{|c|}{ A veces } & \multicolumn{2}{|c|}{ Casi nunca } & \multicolumn{2}{|c|}{ Nunca } & \multirow[t]{2}{*}{ Total } & \multirow[t]{2}{*}{ Atributo } & \multirow[t]{2}{*}{$\sigma 2$} \\
\hline & $\%$ & Peso & $\%$ & Peso & $\%$ & Peso & $\%$ & Peso & $\%$ & Peso & & & \\
\hline 4 & 47,8 & 2,39 & 32,6 & 1,30 & 19,6 & 0,59 & 0,0 & 0,00 & 0,0 & 0,0 & 4,28 & $\begin{array}{c}\text { Muy } \\
\text { Bueno }\end{array}$ & 0,594 \\
\hline 5 & 21,0 & 1,05 & 30,4 & 1,22 & 43,5 & 1,30 & 5,1 & 0,10 & 0,0 & 0,0 & 3,67 & Bueno & 0,741 \\
\hline 6 & 18,1 & 0,91 & 21,0 & 0,84 & 14,5 & 0,43 & 46,4 & 0,93 & 0,0 & 0,0 & 3,11 & Regular & 1,387 \\
\hline & & & & Media & lel inc & icador & & & & & 3,69 & Bueno & 0,230 \\
\hline
\end{tabular}

A diferencia de las preguntas del indicador anterior estas fueron valoradas de manera diferente, obteniendo resultados como Muy bueno, bueno y regular. El indicador tuvo una media de 3.69 por lo que es un valor positivo y una varianza de 0.230 . En las respuestas podemos observar un resultado favorable en cuanto a la mediana empresa, la cual refleja un interés en capacitar a su personal para desarrollar las habilidades, aunque se puede deducir una mayor aceptación por parte del personal en cuanto a la capacitación con recursos propios de la empresa, estos mediante instructores internos que las capacitaciones con recursos externos.

Los resultados enfocados en el indicador de capacidades administrativas, contó en el instrumento con 3 preguntas referentes: (7) ¿La toma de decisiones requiere asumir una postura humana enfocada en desarrollar procedimientos como el reconocimiento del problema, la recolección de información, la determinación del curso a seguir y la verificación de resultados?; 
(8) ¿La empresa promueve el trabajo en equipo, como estrategia para concentrar habilidades, destrezas, conocimientos y responsabilidades?; (9) ¿para lograr los objetivos, la empresa aplica técnicas específicas de motivación?

Los resultados de estas preguntas se muestran en la figura 3 y tabla 3.

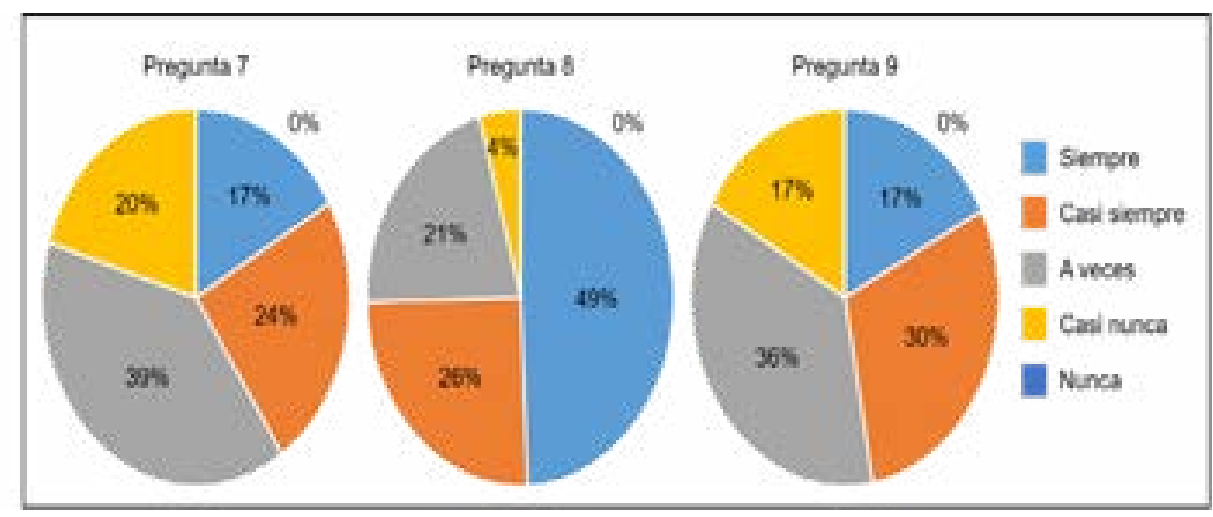

Figura 3. Respuestas de las preguntas 7,8 y 9

Tabla 3. Indicador: capacidades administrativas

\begin{tabular}{|c|c|c|c|c|c|c|c|c|c|c|c|c|c|}
\hline \multirow{2}{*}{ Ítem } & \multicolumn{2}{|c|}{ Siempre } & \multicolumn{2}{|c|}{$\begin{array}{c}\text { Casi } \\
\text { siempre }\end{array}$} & \multicolumn{2}{|c|}{ A veces } & \multicolumn{2}{|c|}{ Casi nunca } & \multicolumn{2}{|c|}{ Nunca } & \multirow{2}{*}{ Total } & \multirow{2}{*}{ Atributo } & \multirow{2}{*}{$\sigma 2$} \\
\hline & $\%$ & Peso & $\%$ & Peso & $\%$ & Peso & $\%$ & Peso & $\%$ & Peso & & & \\
\hline 4 & 16,7 & 0,83 & 23,9 & 0,96 & 39,1 & 1,17 & 20,3 & 0,41 & 0,0 & 0,0 & 3,37 & Regular & 0,972 \\
\hline 5 & 49,3 & 2,46 & 25,4 & 1,01 & 21,0 & 0,63 & 4,3 & 0,09 & 0,0 & 0,0 & 4,20 & Bueno & 0,839 \\
\hline 6 & 17,4 & 0,87 & 29,7 & 1,19 & 36,2 & 1,09 & 16,7 & 0,33 & 0,0 & 0,0 & 3,48 & Bueno & 0,931 \\
\hline \multicolumn{11}{|c|}{ Media del indicador } & 3,68 & Bueno & 0,134 \\
\hline
\end{tabular}

En el presente indicador se obtuvieron 2 calificaciones en bueno y 1 regular, podemos observar resultados no tan satisfactorios a comparación de los anteriores, en este caso la varianza es de 0.134 y la media de medias es de 3.68; este indicador es valorado como bueno. Se puede identificar que el 59\% de las empresas, a veces o casi nunca, toman decisiones metodológicas, no obstante, el $74 \%$ promueve el trabajo entre equipos, como una estrategia de crecimiento, de responsabilidad, de compartir conocimientos y desarrollo de habilidades; y finalmente se puede deducir que el $47 \%$ de estas empresas optan por estrategias de motivación con el fin de incentivar al equipo para alcanzar sus metas. 


\section{CONCLUSIONES}

Con la investigación desarrollada se logró establecer que:

Las medianas empresas de Cúcuta tienden a ser dirigidos por un gerente con formación profesional y contratan al menos un empleado cuyo nivel educativo máximo es el bachillerato.

La mediana empresa en la ciudad de Cúcuta, considera importante la cualificación de su recurso humano para lograr un mejor desempeño y mayor productividad, que le permita a la organización crecer y/o posicionarse en el mercado, adaptándose a cambios y nuevas exigencias.

Se puede deducir que la mediana empresa de Cúcuta manifestó un interés favorable por capacitar a sus empleados, lo cual permitirá desarrollar y potenciar habilidades y conocimientos que serán utilizados en pro del crecimiento de la organización; logrando un mayor desempeño en las actividades de dichos colaboradores, quienes se puede deducir manifestaron el deseo y disposición para capacitarse.

Por último, cabe resaltar que más del $50 \%$ de las medianas empresas Cucuteñas toman decisiones empíricas o no metodológicas, sin embargo, el trabajo en equipo es promovido como una herramienta de intercambio de conocimientos y crecimiento empresarial; situación que puede ser mejorada mediante la implementación de políticas de capacitación y formación profesional.

\section{REFERENCIAS BIBLIOGRÁFICAS}

Arias, F. (2006). El Proyecto de Investigación. Introducción a la Metodología científica. Quinta edición. Caracas: Editorial Episteme

Arocha, J. (2016). Liderazgo Estratégico. Más allá de los hábitos efectivos. Editorial Inver - E - Group Venezuela C.A. Maracaibo Venezuela

Balestrini, M., Diez, T., Marshall, P., Gluhak, A., \& Rogers, Y. (2015). IoT community technologies: leaving users to their own devices or orchestration of engagement? EAI Endorsed Transactions on Internet of Things, 1(1).

Batra, G., \& Tan, H. (2002). Upgrading work force skills to create high-performing firms. Building competitive firms: incentives and capabilities, World Bank Publications: Washington, 118-134.

Bermúdez Carrillo, L. A. (2015). Capacitación: una herramienta de fortalecimiento de las pymes. InterSedes, 16(33), 01-25.

Bernal, C. (2010). Metodología de la investigación. Tercera edición. Pág. 320. Pearson Educación, Colombia, 2010.

Boza, M. (2012). El paradigma de investigación: "La estrella polar del científico". Revista EDUCARE, Volumen 16, Número 1, Enero-Abril 2012 (pp.121-142). [En Línea]. Disponible en: http://revistas.upel.edu.ve/ index.php/educare/article/viewFile/753/256

Cámara de Comercio de Cúcuta. (2014). Informe de gestión 2013. [En línea]. Disponible en: http://www.cccucuta.org.co/media/ Documentos/informe de gestion 2013.pdf

[Chávez, Nilda (2007). Introducción a la Investigación Educativa. Venezuela: Editorial Graficas, S.A 
Colmenares, G y Villasmil, L. (2008). Toma de decisiones y perfil de competencias del gerente de las Instituciones de Educación Superior (ÍES) en Venezuela. En Revista REDHECS URBE Maracaibo. Edición No 5 Año 3 (2008). Disponible en http:// publicaciones.urbe.edu/index.php/ REDHECS/article/view/586/1476

Corbetta, P. (2007). Metodología y técnicas de investigación social. Mcgraw-hill.

Corral, Y. (2009). Validez y confiabilidad de los instrumentos de investigación para la recolección de datos. Revista Ciencias de la Educación. Año 2009. Vol. 19. № 33. p.p 228-247. [En Línea]. Disponible en http:// servicio.bc.uc.edu.ve/educacion/revista/ n33/art12.pdf [Consultado en noviembre 2020]

Gallart, M. A. (2001). La articulación entre el sector público y la empresa privada en la formación profesional de América Latina. En:

Formación para el trabajo: ¿pública o privada?-Montevideo: CINTERFOR, 2001p. 23-59.

Hernández, R; Fernández, C y Baptista, P. (2005). Metodología de la Investigación. Quinta edición: McGraw-Hill Interamericana. México, DF

Mariño, D. (2014). Relación entre motivación laboral y desempeño apasionado.

Marr, B., \& Adams, C. (2004). The balanced scorecard and intangible assets: similar ideas, unaligned concepts. Measuring business excellence.

Martins, F., \& Palella, S. (2006). Metodología de la investigación cuantitativa. FEDUPEL. Caracas. Venezuela.
Méndez, C. (2007). Metodología: diseño y desarrollo del proceso de investigación con énfasis en ciencias empresariales ( $4^{a} \mathrm{Ed}$ ). Editorial LIMUSA

Mezher, Roberto (2014). Estrategias didácticas del docente y su relación con el rendimiento académico estudiantil en la UNERMB. Tesis Doctoral. Universidad Dr. Rafael Belloso Chacín, Doctorado en Ciencias de la Educación, Maracaibo, Venezuela.

Ministerio de Comercio Industria y Turismo de Colombia (MinComercio). (2013). Definición Tamaño Empresarial Micro, Pequeña, Mediana o Grande. [en línea]. Disponible en: http://www.mipymes.gov.co/ publicaciones.php?id=2761 [consultado en noviembre 2020]

Mondy, R. W., \& Noe, R. M. (2005). Administración de recursos humanos. Pearson educación.

Padilla, R., \& Juárez, M. (2006). Efectos de la capacitación en la competitividad de la industria manufacturera. CEPAL.

Palella, S y Martins, F. (2012). Metodología de la investigación cuantitativa 3ra Ed (2012). FEDEUPEL. Caracas. Venezuela

Pérez, D. (2004). El ambiente pensamiento estratégico de la gerencia postmoderna. En Revista CICAG Volumen 3 Edición No 1 - Año 2004 URBE Maracaibo. Disponible en http://publicaciones.urbe.edu/index.php/ cicag/article/view/566/1440

Pulido Polo, M. (2015). Ceremonial y protocolo: métodos y técnicas de investigación científica. 
Ramírez, I. (2009). Los diferentes paradigmas de investigación y su incidencia sobre los diferentes modelos de investigación didáctica. En X. Gabella y P. Reiss (eds.), Seminario de Metodología de la Investigación II.

Ministerio de Educación. Argentina. Disponible en: http://metodologiafeyc.ecaths.com/ ver-trabajos-practicos/2694/orientacion-detrabajo-n-1-diagnostico-grupal-/

Robbins, S; Coulter, M. (2005). Administración. $8^{\mathrm{a}}$ ed. México: Pearson Educación, 2005.

Sánchez, J. (2006). Fundamentos del Trabajo en Equipo para Equipos de trabajo. Editorial McGraw Hill. España.

Supersociedades. Sistema de Información y Reporte Empresarial - SIREM. Consultado en http://www.supersociedades.gov.co/ asuntos-economicos-y-contables/estudiosy-supervision-por-riesgos/SIREM/Paginas/ default.aspx

Tamayo y Tamayo, M. (2003). Proceso de la Investigación Científica. Editorial Noriega Editores. Cuarta Edición. México. 2003

Terán, G. (2006). Paradigmas de Investigación. Concepciones básicas. Papel de Trabajo. Dirección General de Postgrados. Universidad Tecnológica Equinoccial. Ecuador. Disponible en: http://docplayer.es/14360509-Papel-detrabajo-paradigmas-de-investigacionconcepciones-basicas-hacia-unaeducacion-de-calidad-el-proyecto-deinvestigacion-como-elaborar.html

Toro, A \& Marcano, L. (2008). La categoría paradigma en la investigación social. Disponible en https://www.saber.ula.ve/ bitstream/123456789/21020/1/articulo1.pdf 